\title{
Vonoprazan-Based Triple-Therapy Could Improve Efficacy of the Tailored Therapy of Helicobacter pylori Infection
}

\author{
Tetsuya Shinmura, Kazunori Adachi, Yoshiharu Yamaguchi, Shinya Izawa, Yasutaka Hijikata, Masahide Ebi, Yasushi \\ Funaki, Naotaka Ogasawara, Makoto Sasaki, Kunio Kasugai
}

Division of Gastroenterology,

Department of Internal

Medicine, Aichi Medical

University School of Medicine,

Nagakute, Japan

\section{Address for correspondence:}

Makoto Sasaki

Division of Gastroenterology,

Department of Internal

Medicine

Aichi Medical University

School of Medicine

1-1 Yazakokarimata, Nagakute,

Aichi, Japan, 480-1195

msasaki@aichie-med-u.ac.jp
Received: 25.06 .2019

Accepted: 25.07.2019

\section{ABSTRACT}

Background \& Aims: The prevalence of clarithromycin resistant bacteria is increasing, and the effectiveness of Helicobacter pylori (H. pylori) triple therapy is gradually decreasing in Japan. Vonoprazan, a potassiumcompetitive acid blocker, has been reported for its effectiveness in eradicating $H$. pylori. We aimed to evaluate the efficacy of tailored vonoprazan-based triple therapy in patients with $H$. pylori. This study is the first to compare the efficacy of vonoprazan-based tailored triple therapy to that of vonoprazan-based conventional therapy.

Method: This retrospective cohort study evaluated the treatment efficacy in 920 patients. Of these, 541 received conventional and 379 received tailored therapy. Successful eradication was confirmed by a negative ${ }^{13} \mathrm{C}$-urea breath test 6-8 weeks following completion of $H$. pylori eradication therapy, and the data were evaluated using the Chi-square test, or Fisher's exact test, as appropriate.

Results: The eradication rate of tailored therapy was $90 \%$ and $96.3 \%$ by intent-to-treat analysis and per protocol analysis, respectively, which was significantly higher than the $85 \%$ and $90.2 \%$ found for conventional therapy ( $\mathrm{p}<0.05$ and $\mathrm{p}<0.001$, respectively). Amoxicillin- or clarithromycin-resistant bacteria did not affect treatment outcomes. By univariate and multivariate analysis, both amoxicillin- and clarithromycin-resistant bacteria and conventional therapy were detected as risk factors for eradication failure (odds ratio $=6.267$, 95\% CI [1.056-119.924], $\mathrm{p}<0.05$, and odd ratio $=3.113,95 \%$ confidence interval [1.688-6.160], $\mathrm{p}<0.001$, by multivariate analysis).

Conclusion: Vonoprazan-based triple therapy could be a more effective treatment for $H$. pylori infection than conventional therapy when combined with a therapy regimen tailored according to bacterial antibiotic susceptibility.

Key words: Helicobacter pylori - conventional therapy - clarithromycin - amoxicillin - drug resistance microbial drug resistance - vonoprazan.

Abbreviations: AMX: amoxicillin; AMX-R: amoxicillin resistant; AMX-S: amoxicillin sensitive; CAM: clarithromycin; CAM-R: clarithromycin resistant; CAM-S: clarithromycin sensitive; H. pylori: Helicobacter pylori; ITT: intent-to-treat; MNZ: metronidazole; PPI: proton pump inhibitor; PPS: per protocol set; VPZ: vonoprazan.

\section{INTRODUCTION}

Helicobacter pylori $(H$. pylori) is one of the most prevalent bacterial pathogens and is associated with upper gastrointestinal disorders such as gastritis, peptic ulcer, functional dyspepsia, gastric mucosaassociated lymphoid tissue lymphoma, and gastric cancer [1-3]. Eradication of $H$. pylori infection provides an effective approach for curing or preventing the associated diseases [4-6]. However, the effectiveness of the most commonly recommended first-line regimen has declined.

The treatment success of $H$. pylori involves many factors, including point mutation conferring antibiotic resistance and the genetic polymorphisms influencing drug metabolism, which vary by the geographic area $[7,8]$. Therefore, the same regimen may be extremely effective in one geographic area but ineffective in another. The Japanese Society for Helicobacter Research recommends a 7-day triple therapy using a proton pump inhibitor (PPI) or vonoprazan (VPZ) with amoxicillin (AMX) and clarithromycin (CAM) [9]; this is the only firstline regimen currently covered by the Japanese national health 
insurance system. In the rescue therapy, a 7-day triple therapy using PPI or VPZ with AMX and metronidazole (MNZ) is the only second-line regimen currently covered by the Japanese national health insurance system. The resistance rate of MNZ in Japan is very low (from $1.1 \%$ to $2.5 \%$ ) [10]; therefore $99 \%$ of patients with $H$. pylori infection are cured by first- and second-line regimens. In Japan, CAM-based 7-day triple therapy was approved for gastric and duodenal ulcers in 2000, when the initial eradication rate was approximately 90\% [11] However, the eradication rate of triple therapy has decreased with the increase of CAM resistant (CAM-R) strains [12]. The Maastricht IV/Florence Consensus Report recommended that anti-H. pylori regimen should consider the local CAM resistance rate, with regions of high or low resistance identified using a $15-20 \%$ threshold [13]. A working group from the Japanese Society of Helicobacter Research performed a surveillance study to determine the temporal antimicrobial susceptibility profiles of $H$. pylori isolated during 2002-2006 and 2010-2011 in 67 institutions; this resulted in the analysis of 7,735 isolates. Primary CAM-R increased from $19 \%$ to $31 \%$ as CAM use increased; therefore, CAM treatment should not be used without an antibiotic susceptibility test.

Recently, VPZ, a novel potassium-competitive acid blocker, has been used in H. pylori eradication therapy in order to increase the eradication rate $[14,15]$. Our previous study demonstrated that the first-line eradication rate of VPZ-based triple therapy was $89.7 \%$, significantly higher than that of PPI-based triple therapy in per protocol set (PPS) analysis. Additionally, even in patients with CAM-R bacteria, the eradication rate was significantly higher for those treated with VPZ compared to those treated with a PPI (87.5\% vs. $53.8 \%)$ [14]. However, concerns related to the CAM-R strains remain, because this eradication rate failed to achieve an acceptable grade according to the report card for grading $H$. pylori therapies proposed by Graham [16]. It has been reported that tailoring therapy according to bacterial CAM susceptibility can improve the eradication rate in PPI-based triple therapy, but no study has examined the efficacy of VPZ-based triple therapy. Additionally, the influence of bacterial amoxicillin (AMX) susceptibility in VPZ-based triple therapy is unclear.

This study aimed to find out whether tailoring $H$. pylori treatment according to CAM susceptibility could improve the eradication rate in VPZ-based triple therapy, and to assess the influence of bacterial AMX susceptibility in eradication therapy.

\section{METHODS}

\section{Statement of ethics}

This study conformed to the code of ethics stated in the Declaration of Helsinki. The research protocols were approved by the appropriate Ethics Committee (approval number 2018H057).

\section{Study population}

This was a retrospective cohort study. All patients who had an initial $H$. pylori infection treatment from October 2015 to December 2018 were enrolled. Patients who underwent PPIbased triple therapy, a MNZ-containing regimen without an antibiotic susceptibility test, a MNZ-based regimen against CAM sensitive (CAM-S) H. pylori or had both CAM-R and MNZ resistant (MNZ-R) bacteria were excluded from the study.

\section{H. pylori eradication therapies}

The treatment regimen selection was dependent upon the attending doctor. Basically, the patients who did not undergo a bacterial susceptibility test by esophagogastroduodenoendoscopic examination received conventional therapy (VPZ-based CAM-containing 7-day triple therapy: $20 \mathrm{mg}$ VPZ, twice daily (b.i.d.); $750 \mathrm{mg}$ AMX, b.i.d.; and $200 \mathrm{mg}$ CAM, b.i.d.). The patients who did not yet have a bacterial susceptibility test result prior to the start of treatment also received conventional therapy. Patients who already had a bacterial susceptibility test result prior to starting treatment received tailored therapy (VPZ-based CAM-containing 7-day triple therapy: $20 \mathrm{mg}$ VPZ, b.i.d.; 750 mg AMX, b.i.d.; and $200 \mathrm{mg}$ CAM, b.i.d. in the case of CAM-S bacteria and VPZ-based MNZ-containing 7-day triple therapy: 20 mg VPZ, b.i.d.; 750 mg AMX, b.i.d.; and 250 mg MNZ, b.i.d. in the case of CAM-R bacteria).

\section{Diagnosis of $\boldsymbol{H}$. pylori infection}

Positive diagnosis for $H$. pylori infection was defined as endoscopic active gastritis and positivity in one of four tests (rapid urease test, histology, serum antibody, or culture). Successful eradication was confirmed by a negative ${ }^{13} \mathrm{C}$-urea breath test $6-8$ weeks following completion of $H$. pylori eradication therapy.

\section{H. pylori susceptibility to amoxicillin, clarithromycin,} and metronidazole

H. pylori isolates were obtained from gastric biopsy specimens before treatment. AMX-R, CAM-R, and MNZ-R were defined using the flat dilution method as a minimum inhibitory concentration of $\geq 0.06 \mu \mathrm{g} / \mathrm{ml}, \geq 1 \mu \mathrm{g} / \mathrm{ml}$, and $\geq 16$ $\mu \mathrm{g} / \mathrm{ml}$ for $H$. pylori, respectively.

\section{Statistical analyses}

The results are presented as the mean \pm standard division (SD), and as numbers and percentages. The quantitative data were analyzed using the $t$-test, and categorical data were analyzed using the Chi-squared test or Fisher's exact test, as appropriate. Logistic regression was used to obtain the eradication failure odds ratio. The variable was included in the multivariate analysis when a $\mathrm{p}$-value $<0.2$ was observed in the univariate analysis. All data were analyzed using JMP ${ }^{\circ}$ Version 14.2 (SAS Institute Inc., Cary, NC, USA). A p-value < 0.05 was considered significant.

\section{RESULTS}

A total of 1,001 patients were candidates for this study. Eighty-one patients were excluded: 52 underwent PPI-based triple therapy, 14 underwent an MNZ-containing regimen without an antibiotic susceptibility test, 9 underwent an MNZ-containing regimen against CAM-S bacteria, and 6 had both CAM-R and MNZ-R bacteria. A total of 541 patients 


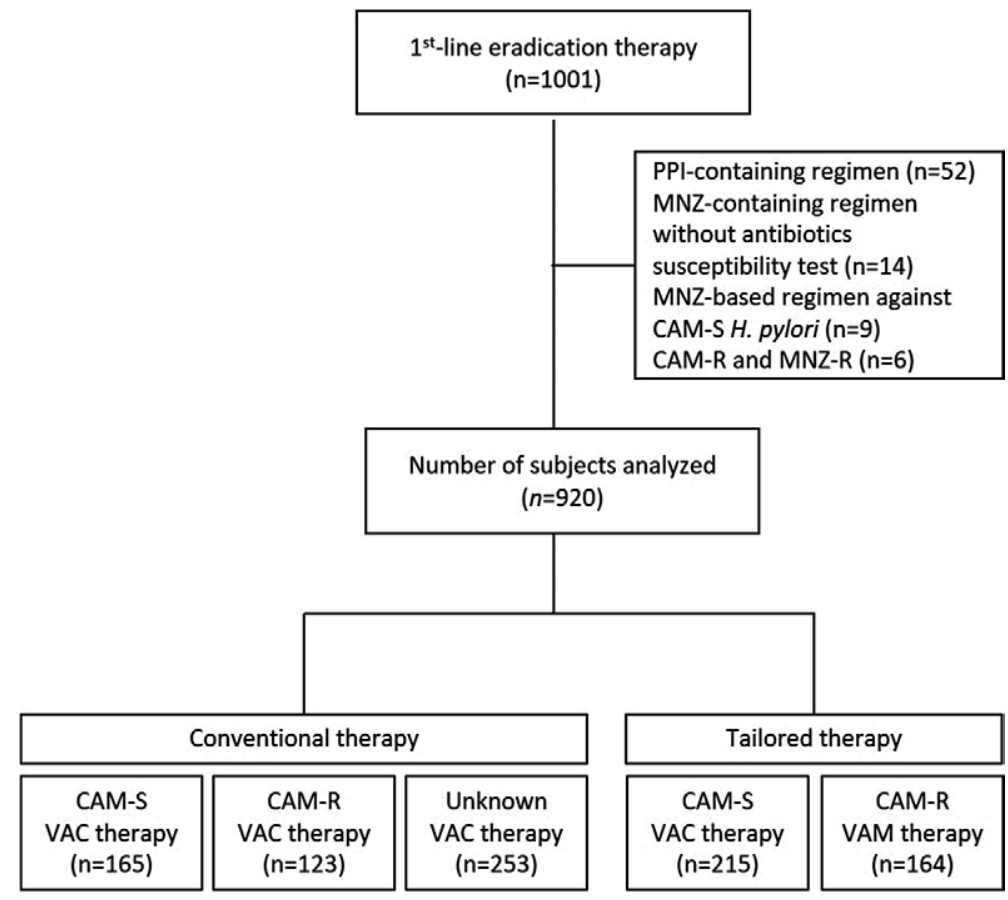

Fig. 1. Patient enrolment flow chart.

received conventional therapy: 165 had CAM-S bacteria and 123 had CAM-R bacteria. A total of 379 patients received tailored therapy: 215 had CAM-S bacteria and received CAM-containing triple therapy, and 164 had CAM-R bacteria and received MNZ-containing triple therapy (Fig. 1). Patient characteristics are shown in Table I. There were no differences in patient characteristics between the conventional and tailored therapy groups. The resistance rates of AMX and CAM were $14.4 \%$ and $43.0 \%$, respectively.

The eradication rate of tailored therapy by intent-to-treat (ITT) analysis and PPS analysis was $90.0 \%$ (95\% confidence interval - CI 86.5\%-92.6\%) and 96.3\% (95\%CI 93.8\%-97.8\%), respectively, which was significantly higher than that of conventional therapy: $85.0 \%$ (95\%CI $81.8 \%-87.8 \%)$ and $90.2 \%$ (95\%CI 87.4\%-92.5\%), $\mathrm{p}=0.0028$ and $\mathrm{p}=0.0008$, respectively (Fig. 2).

In the conventional therapy group, the eradication rate of patients with CAM-R bacteria $(85.8 \%$, 95\%CI 78.5\%-91.0\%) was significantly lower than in those with CAM-S (93.2\%, 95\%CI 88.2\%-96.1\%) $(\mathrm{p}=0.046)$. In the tailored therapy group, the eradication rate of patients with CAM-R bacteria (98.1\%, 95\%CI 94.4\%-99.3\%) did not differ from those with CAM-S bacteria (95.0\%, 95\% CI 91.0\%-97.3\%), and was significantly increased compared to those with CAM-R bacteria in the

Table I. Characteristics of patients who received first-line vonoprazan-based triple therapy for H. pylori.

\begin{tabular}{|c|c|c|c|c|c|}
\hline & \multicolumn{2}{|c|}{$\begin{array}{l}\text { Conventional therapy } \\
\mathrm{n}=541\end{array}$} & \multicolumn{2}{|c|}{$\begin{array}{l}\text { Tailored therapy } \\
\mathrm{n}=379\end{array}$} & $\mathrm{p}$ value \\
\hline \multicolumn{6}{|l|}{ Gender } \\
\hline Female, $\mathrm{n}$ & 250 & $(46.2 \%)$ & 176 & $(46.4 \%)$ & 0.947 \\
\hline Male, $\mathrm{n}$ & 291 & $(53.8 \%)$ & 203 & $(53.6 \%)$ & \\
\hline Age, years & $62.5 \pm 13.9$ & $63.8 \pm 12.6$ & & & 0.129 \\
\hline \multicolumn{6}{|l|}{ Diagnosis } \\
\hline Gastritis, $\mathrm{n}$ & 444 & $(82.1 \%)$ & 316 & $(83.4 \%)$ & 0.846 \\
\hline Peptic ulcer, n & 58 & $(10.7 \%)$ & 39 & $(10.3 \%)$ & \\
\hline Others, $\mathrm{n}$ & 39 & $(7.2 \%)$ & 24 & $(6.3 \%)$ & \\
\hline \multicolumn{6}{|l|}{ AMX susceptibility } \\
\hline sensitive, $\mathrm{n}$ & 255 & $(88.5 \%)$ & 316 & $(83.4 \%)$ & 0.074 \\
\hline resistant, $\mathrm{n}$ & 33 & $(11.5 \%)$ & 63 & $(16.6 \%)$ & \\
\hline \multicolumn{6}{|l|}{ CAM susceptibility } \\
\hline sensitive, $\mathrm{n}$ & 165 & $(57.3 \%)$ & 215 & $(56.7 \%)$ & 0.937 \\
\hline resistant, $\mathrm{n}$ & 123 & $(42.7 \%)$ & 164 & $(43.3 \%)$ & \\
\hline
\end{tabular}

AMX: amoxicillin, CAM: clarithromycin 
conventional therapy group $(\mathrm{p}=0.0002)$. In patients with CAM-S bacteria, there was no difference in the eradication rate between conventional and tailored therapies.

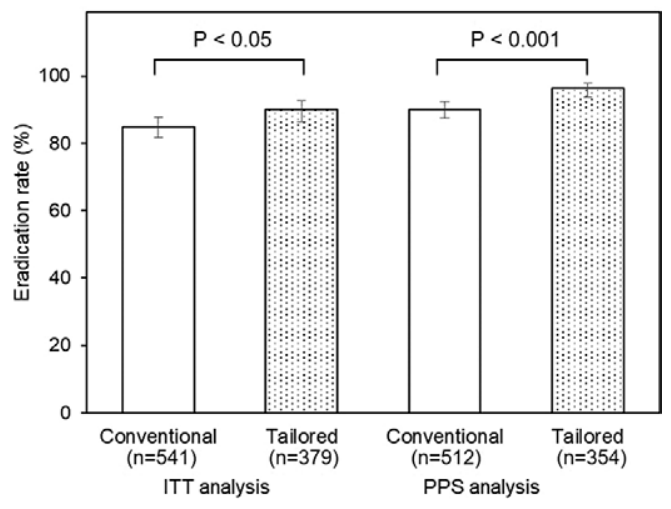

Fig. 2. Comparison of $H$. pylori eradication rate between conventional therapy and tailored therapy. By ITT analysis, the eradication rate of tailored therapy was $90.0 \%$, which was increased compared to $85.0 \%$ of conventional therapy $(\mathrm{p}<0.05)$. By PPS analysis, the eradication rate of tailored therapy was increased compared to conventional therapy ( $96.3 \%$ vs $90.2 \%, \mathrm{p}<0.001)$.

In the conventional therapy group, the eradication rate of the patients with CAM-S and AMX-R bacteria, and CAM-R and AMX-S bacteria was $92.3 \%$ (95\%CI 66.7\%-98.6\%) and 90.0\% (95\%CI 82.6\%-94.5\%), respectively, which did not differ from the $93.2 \%$ (95\%CI 88.0\%-96.3\%) of patients with CAM-S and AMX-S bacteria. The eradication rate of patients with CAM-R and AMX-R bacteria (65.0\%, 95\%CI 43.3\%-81.9\%) was significantly lower than that of patients with CAM-S and AMX-S bacteria $(\mathrm{p}=0.001)$. However, in the tailored therapy group, bacterial antibiotic susceptibility had no influence on the eradication rate (Fig. 3).

By univariate analysis, having both AMX-R and CMA-R, and conventional therapy was detected as a risk factor for eradication failure ( $\mathrm{p}=0.017$, and $\mathrm{p}=0.0008$, respectively) (Table II). By multivariate analysis using the factors with $\mathrm{p}<0.2$ by univariate analysis, AMX-R and CAM-R, and conventional therapy were detected as risk factors for the failure of VPZbased triple therapy $(\mathrm{p}=0.042$ and $\mathrm{p}=0.0005$, respectively) (Table II).

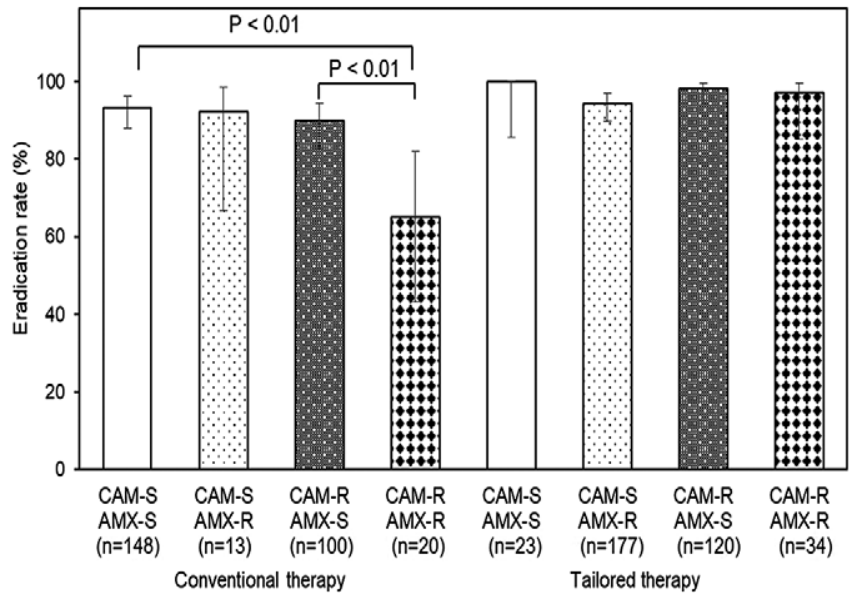

Fig. 3. Comparison of the $H$. pylori eradication rate according to antibiotic susceptibility of bacteria. In the conventional therapy, the eradication rate for both CAM-R and AMX-R was significantly decreased compared to CAM-R $(\mathrm{p}<0.01)$ and antibiotics sensitive $(\mathrm{p}<0.05)$.

\section{DISCUSSION}

In the present study, tailored therapy showed the best eradication rate $(90.0 \%)$ by ITT analysis, which reached an acceptable grade $\mathrm{B}$ according to the report card for grading $H$. pylori therapies proposed by Graham [16]. As far as we know, this is the first report comparing the efficacy of VPZ-based tailored triple therapy to VPZ-based conventional therapy.

Antibiotic susceptibility, especially CAM susceptibility, has been attracting attention regarding its role in the triple therapy success of $H$. pylori eradication because the efficacy of regimens containing CAM is greatly affected by CAM resistance [12].

In 2000, the resistance rate for CAM was $7.0 \%$; however, while it increased to $27.7 \%$ in 2004 [10, 17], the eradication rate has inversely decreased. In this study the resistance rates of AMX and CAM were $14.4 \%$ and $43.0 \%$, respectively; these rates are the same as those reported previously $[10,18]$. Our previous study showed that a VPZ-based CAM-containing triple regimen achieved a higher eradication rate than a PPIbased CAM-containing triple regimen in first-line eradication therapy [14]. VPZ-containing therapy was more effective than PPI-containing therapy for patients with CAM-R bacteria

Table II. Risk factors for eradication failure.

\begin{tabular}{lcccccc}
\hline & \multicolumn{3}{c}{ Univariate analysis } & \multicolumn{3}{c}{ Multivariate analysis } \\
\hline Factor & OR & $95 \%$ CI & p value & OR & $95 \%$ CI & p value \\
\hline Female & 0.973 & {$[0.582-1.628]$} & 0.919 & & & \\
Age $\geq 60$ & 0.998 & {$[0.583-1.706]$} & 0.993 & & & \\
Gastritis & 1.090 & {$[0.541-2.196]$} & 0.809 & & & \\
AMX-R & 1.781 & {$[0.820-3.869]$} & 0.139 & 0.497 & {$[0.026-2.424]$} & 0.450 \\
CAM-R & 1.275 & {$[0.677-2.402]$} & 0.452 & & & \\
AMX-R+CAM-R & 2.608 & {$[1.269-5.360]$} & 0.017 & 6.267 & {$[1.056-119.924]$} & 0.042 \\
Conv. therapy & 2.839 & {$[1.518-5.309]$} & 0.0008 & 3.113 & {$[1.688-6.160]$} & 0.0005 \\
\hline
\end{tabular}

OR: odd ratio, CI: confidence interval, AMX-R: amoxicillin resistance, CAM-R: clarithromycin resistance, Conv.: conventional 
in randomized controlled trials [19]. Furthermore, a recent meta-analysis showed the advantage of VPZ use compared to PPI [20]. Similar to previous reports $[15,20]$, the eradication rate of conventional therapy in this study was $85.0 \%$ by ITT analysis, suggesting the long-lasting stability of the increased eradication rate from VPZ-based triple therapy, even in areas where the CAM-R bacteria rate was over $40 \%$. This high eradication rate in VPZ-based triple therapy has been attributed to a rapid and prolonged (24-h) acid suppression ( $\mathrm{pH}>4$, holding time ratio $100 \%)$ [21], which affects $H$. pylori eradication $[22,23]$. Unlike PPI, which is activated by acid to function, VPZ inhibits potassium intake and the subsequent release of hydrogen ions by selectively binding to parietal cells [24-27]. However, no report has investigated the advantage provided by VPZ in direct use and acid inhibition during VPZ based eradication therapy. While the eradication rate of regimens containing AMX is affected by AMX-R [28], the effect of bacterial AMX susceptibility in VPZ-based triple therapy has never been considered. In this study, the effect of AMX and CAM antibiotic susceptibility were analyzed in terms of eradication success. The eradication rate of only AMX or CAM resistant bacteria did not differ from that of non-antibiotic resistant bacteria. The eradication rate in patients with both AMX and CAM resistant bacteria in conventional therapy was extremely decreased compared to those with single strain or non-antibiotic resistant bacteria. Combined resistance to AMX and CAM, but not AMX or CAM resistance alone, was a significant risk factor for eradication failure in VPZ-based triple therapy. The AMX resistance rate in our study was $14.4 \%$, higher than the 5.5\% reported in 2004 [10], and suggests an increasing AMX resistance rate. Hereafter, the eradication rate of VPZ-based triple therapy might decrease owing to the increasing number of AMX and CAM resistant bacteria.

The main reason for eradication failure is the rapid increase in antibiotic resistance of bacteria, poor patient compliance, and the rapid metabolism of conventional PPIs. Additionally, there are geographic differences in bacterial antibiotic resistance and genotype in the rapid metabolism of conventional PPIs. The frequency of AMX resistance is quite low worldwide, except in Cameroon, Iran, and Japan [10, 29, 30]. In eastern countries, the frequency of the A2143G mutation, which has a much stronger impact on conferring antibiotic resistance, is lower than that in western countries. The frequency of poor CYP2C19 metabolizers is higher in the Japanese population compared to the Caucasian populations $[7,8]$. Therefore, it is necessary to consider the geographic area where the trial was performed when interpreting research results.

Before VPZ appeared on the market, a study on a tailored treatment regimen based on CAM susceptibility was performed to improve the $H$. pylori eradication rate, and achieved approximately $95 \%$ by PPI-based triple therapy [31, 32]. In our study, the efficacy of tailored VPZ-based triple therapy was shown for the first time. Tailored therapy significantly increased the eradication rate from $85.0 \%$ to $90.2 \%$ by ITT analysis, and up-graded the treatment from grade $\mathrm{C}$ (acceptable) to grade $\mathrm{B}$ (good) based on Graham's reporting system [16]. Per-protocol set analysis indicated the tailored therapy eradication rate was $96.3 \%$, which is considered 'excellent' by the report card system [16].
The eradication rate of conventional triple therapy was improved by the use of VPZ instead of PPI. However, the eradication rate of patients with CAM-R bacteria ranged from $73.2 \%$ to $87.5 \%$ [15], which is an unacceptable level. In this study, the reason for $H$. pylori eradication failure in VPZ-based triple therapy was elucidated for the first time. As antibiotics are widely used, combined AMX and CAM resistant bacteria continue to increase. Therefore, the eradication rate of VPZ-based conventional therapy may decrease in the future, as the eradication rate of PPI-based conventional therapy decreased over the past decades [12]. Tailored therapy could be the best option to achieve an optimal eradication result. Heteroresistance (simultaneous presence of both susceptible and resistant strains at different sites in the same stomach) should be considered in patients with pangastritis, because $H$. pylori is not uniformly distributed in the stomach [33]. To avoid this problem, two biopsy specimens were obtained from the antrum and corpus for the antibiotic susceptibility examination in this study. This might be the reason for the improved eradication rate, even in the VPZ-based triple therapy.

Our results must be interpreted within the limitations of our study. First, the study design was a retrospective singlecenter cohort study. Antibiotic resistance related data should be considered in light of the varying responses obtained in different geographic areas, even those within the same district. A worldwide, multicenter, prospective randomized trial is necessary to show the superiority of tailored therapy. Nonetheless, it is impossible to perform trials examining VPZ efficacy in $H$. pylori infected populations in countries other than Japan. Second, eradication success might be influenced by several factors such as antibiotic susceptibility, insufficient acid inhibition, bacterial genotypes that reduce virulence, and compliance $[34,35]$, but bacterial genotypes and compliance could not be analyzed. Tailored therapy effectiveness measured by molecular tests has achieved higher success rates than that measured by traditional culture-based tests [36]. Further research is necessary to compare the eradication rate of tailored therapy by both molecular and culture testing.

\section{CONCLUSION}

Vonoprazan improved the eradication rate of the first-line triple-therapy. In addition, VPZ could improve even a tailored therapy based on the antibiotic susceptibility of bacteria.

Conflicts of interest: M.S. received a joint research grant from Amano Enzyme Inc., and K.K. received lecture fees from Daiichi Sankyo Co., Ltd., Astra Zeneca Co., Ltd., EA Pharma Co., Ltd., Mylan Co., Ltd., Sanwa Kagaku Kenkyusho Co., Ltd., and Takeda Pharmaceutical Co., Ltd. K.K. received research grants from Astellas Co., Ltd., Daiichi Sankyo Co., Ltd., EA Pharma Co., Ltd., Mitsubishi Tanabe Pharma Co., Ltd., and Takeda Pharmaceutical Co., Ltd. The other authors have no conflicts of interest to declare.

Authors' contribution: M.S. concept and design of; T.S., K.A., Y.Y., S.I., Y. H., M.E., Y.F., N.O., and K.K. data acquisition; M.S. statistical analysis. T.S. analyzed the data and drafted the manuscript. All authors critically revised the manuscript, approved the final version to be published, and agree to be accountable for all aspects of the work. 
Acknowledgement. We would like to thank Editage (www.editage. jp) for the English language editing.

\section{REFERENCES}

1. Suzuki H, Hibi T, Marshall BJ. Helicobacter pylori: present status and future prospects in Japan. J Gastroenterol 2007;42:1-15. doi: 10.1007/ s00535-006-1990-Z

2. Cover TL, Blaser MJ. Helicobacter pylori in health and disease. Gastroenterology 2009;136:1863-1873. doi:10.1053/j.gastro.2009.01.073

3. Suzuki H, Moayyedi P. Helicobacter pylori infection in functiona dyspepsia. Nat Rev Gastroenterol Hepatol 2013;10:168-174. doi:10.1038/nrgastro.2013.9

4. Fukase K, Kato M, Kikuchi S, et al. Effect of eradication of Helicobacter pylori on incidence of metachronous gastric carcinoma after endoscopic resection of early gastric cancer: an open-label, randomised controlled trial. Lancet 2008;372:392-397. doi:10.1016/S0140-6736(08)61159-9

5. Suzuki H, Nishizawa T, Hibi T. Helicobacter pylori eradication therapy. Future Microbiol 2010;5:639-648. doi:10.2217/fmb.10.25

6. Kawamura Y, Funaki Y, Yoshimine T, et al. Characteristics and Predictive Factor of Helicobacter pylori-Associated Functional Dyspepsia in Japanese Patients. Digestion 2019:1-9. doi:10.1159/000496101

7. Ierardi E, Giorgio F, Losurdo G, Di Leo A, Principi M. How antibiotic resistances could change Helicobacter pylori treatment: A matter of geography? World J Gastroenterol 2013;19:8168-8180. doi:10.3748/ wjg.v19.i45.8168

8. Goldstein JA, Ishizaki T, Chiba K, et al. Frequencies of the defective CYP2C19 alleles responsible for the mephenytoin poor metabolizer phenotype in various Oriental, Caucasian, Saudi Arabian and American black populations. Pharmacogenetics 1997;7:59-64.

9. Asaka M, Kato M, Takahashi S, et al. Guidelines for the management of Helicobacter pylori infection in Japan: 2009 revised edition. Helicobacter 2010;15:1-20. doi:10.1111/j.1523-5378.2009.00738.x

10. Kobayashi I, Murakami K, Kato M, et al. Changing antimicrobia susceptibility epidemiology of Helicobacter pylori strains in Japan between 2002 and 2005. J Clin Microbiol 2007;45:4006-4010. doi:10.1128/JCM.00740-07

11. Asaka M, Sugiyama T, Kato M, et al. A multicenter, double-blind study on triple therapy with lansoprazole, amoxicillin and clarithromycin for eradication of Helicobacter pylori in Japanese peptic ulcer patients. Helicobacter 2001;6:254-261. doi:10.1046/j.1523-5378.2001.00037.x

12. Sasaki M, Ogasawara N, Utsumi K, et al. Changes in 12-Year FirstLine Eradication Rate of Helicobacter pylori Based on Triple Therapy with Proton Pump Inhibitor, Amoxicillin and Clarithromycin. J Clin Biochem Nutr 2010;47:53-58. doi:10.3164/jcbn.10-10

13. Malfertheiner P, Megraud F, O'Morain CA, et al. Management of Helicobacter pylori infection--the Maastricht IV/ Florence Consensus Report. Gut 2012;61:646-664. doi:10.1136/gutjnl-2012-302084

14. Noda H, Noguchi S, Yoshimine T, et al. A Novel Potassium-Competitive Acid Blocker Improves the Efficacy of Clarithromycin-containing 7-day Triple Therapy against Helicobacter pylori. J Gastrointestin Liver Dis 2016;25:283-288. doi:10.15403/jgld.2014.1121.253.7hp

15. Sugimoto M, Yamaoka Y. Role of Vonoprazan in Helicobacter pylori Eradication Therapy in Japan. Front Pharmacol 2018;9:1560 doi:10.3389/fphar.2018.01560

16. Graham DY, Lu H, Yamaoka Y. A report card to grade Helicobacter pylori therapy. Helicobacter 2007;12: 275-278. doi:10.1111/j.15235378.2007.00518.x
17. Kato M, Yamaoka Y, Kim JJ, et al. Regional differences in metronidazole resistance and increasing clarithromycin resistance among Helicobacter pylori isolates from Japan. Antimicrob Agents Chemother 2000;44:22142216. doi:10.1128/aac.44.8.2214-2216.2000

18. Okamura T, Suga T, Nagaya T, et al. Antimicrobial resistance and characteristics of eradication therapy of Helicobacter pylori in Japan: a multi-generational comparison. Helicobacter 2014;19:214-220. doi:10.1111/hel.12124

19. Li M, Oshima T, Horikawa T, et al. Systematic review with meta-analysis: Vonoprazan, a potent acid blocker, is superior to proton-pump inhibitors for eradication of clarithromycin-resistant strains of Helicobacter pylori. Helicobacter 2018;23: e12495. doi:10.1111/hel.12495

20. Jung YS, Kim EH, Park CH. Systematic review with meta-analysis: the efficacy of vonoprazan-based triple therapy on Helicobacter pylori eradication. Aliment Pharmacol Ther 2017;46:106-114. doi:10.1111/ apt.14130

21. Kagami T, Sahara S, Ichikawa $H$, et al. Potent acid inhibition by vonoprazan in comparison with esomeprazole, with reference to CYP2C19 genotype. Aliment Pharmacol Ther 2016;43:1048-1059. doi:10.1111/apt.13588

22. Sugimoto M, Furuta T, Shirai N, et al. Evidence that the degree and duration of acid suppression are related to Helicobacter pylori eradication by triple therapy. Helicobacter 2007;12:317-323. doi:10.1111/j.1523-5378.2007.00508.x

23. Echizen H. The First-in-Class Potassium-Competitive Acid Blocker, Vonoprazan Fumarate: Pharmacokinetic and Pharmacodynamic Considerations. Clin Pharmacokinet 2016;55:409-418. doi:10.1007/ s40262-015-0326-7

24. Shin JM, Inatomi N, Munson K, et al. Characterization of a novel potassium-competitive acid blocker of the gastric H,K-ATPase, 1-[5-(2-fluorophenyl)-1-(pyridin-3-ylsulfonyl)-1H-pyrrol-3-yl]-Nmethylmethanamine monofumarate (TAK-438). J Pharmacol Exp Ther 2011;339:412-420. doi:10.1124/jpet.111.185314

25. Matsukawa J, Inatomi N, Otake K. Pharmacological and clinical profiles of a novel potassium-competitive acid blocker, vonoprazan fumarate (Takecab((R)) $10 \mathrm{mg}$ and $20 \mathrm{mg}$ ). Nihon Yakurigaku Zasshi 2015;146:275-282. doi:10.1254/fpj.146.275

26. Sakurai Y, Nishimura A, Kennedy G, et al. Safety, Tolerability, Pharmacokinetics, and Pharmacodynamics of Single Rising TAK-438 (Vonoprazan) Doses in Healthy Male Japanese/non-Japanese Subjects. Clin Transl Gastroenterol 2015;6:e94. doi:10.1038/ctg.2015.18

27. Scott DR, Munson KB, Marcus EA, Lambrecht NW, Sachs G. The binding selectivity of vonoprazan (TAK-438) to the gastric H+, K+ -ATPase. Aliment Pharmacol Ther 2015;42:1315-1326. doi:10.1111/apt.13414

28. Chen MJ, Wu MS, Chen CC, et al. Impact of amoxicillin resistance on the efficacy of amoxicillin-containing regimens for Helicobacter pylori eradication: analysis of five randomized trials. J Antimicrob Chemother 2017;72:3481-3489. doi:10.1093/jac/dkx320

29. De Francesco V, Giorgio F, Hassan C, et al. Worldwide H. pylori antibiotic resistance: a systematic review. J Gastrointestin Liver Dis 2010;19:409-414.

30. Milani M, Ghotaslou R, Akhi MT, et al. The status of antimicrobia resistance of Helicobacter pylori in Eastern Azerbaijan, Iran: comparative study according to demographics. J Infect Chemother 2012;18:848-852. doi:10.1007/s10156-012-0425-4

31. Kawai T, Yamagishi T, Yagi K, et al. Tailored eradication therapy based on fecal Helicobacter pylori clarithromycin sensitivities. J Gastroenterol Hepatol 2008;23 Suppl 2: S171-S174. doi:10.1111/j.14401746.2008.05408.x 
32. Sugimoto M, Uotani T, Sahara S, et al. Efficacy of tailored Helicobacter pylori eradication treatment based on clarithromycin susceptibility and maintenance of acid secretion. Helicobacter 2014;19:312-318. doi:10.1111/hel.12128

33. Mascellino MT, Porowska B, De Angelis M, Oliva A. Antibiotic susceptibility, heteroresistance, and updated treatment strategies in Helicobacter pylori infection. Drug Des Devel Ther 2017;11:2209-2220. doi:10.2147/DDDT.S136240

34. Kuo YT, Liou JM, El-Omar EM, et al. Primary antibiotic resistance in Helicobacter pylori in the Asia-Pacific region: a systematic review and meta-analysis. Lancet Gastroenterol Hepatol 2017;2:707-715 doi:10.1016/S2468-1253(17)30219-4

35. Gerrits MM, van Vliet AH, Kuipers EJ, Kusters JG. Helicobacter pylori and antimicrobial resistance: molecular mechanisms and clinical implications. Lancet Infect Dis 2006;6:699-709. doi:10.1016/S14733099(06)70627-2

36. Chen H, Dang Y, Zhou X, Liu B, Liu S, Zhang G. Tailored Therapy Versus Empiric Chosen Treatment for Helicobacter pylori Eradication: A Meta-Analysis. Medicine (Baltimore) 2016;95:e2750. doi:10.1097/ MD.0000000000002750 